\title{
AVALIAÇÃO DA QUALIDADE DE VIDA EM PACIENTES CIRRÓTICOS COM HÉRNIA DA PAREDE ABDOMINAL
}

\section{Quality of life in patients with abdominal hernia and cirrhosis}

\author{
Wellington ANDRAUS, Bianca PAOLETTI, Rafael Soares Nunes PINHEIRO, \\ Fabricio Souza BITENCOURT, Cynthia N. de Freitas FARIAS, Luiz Augusto Carneiro D'ALBUQUERQUE
}

ABCDDV/677

Andraus W, Paoletti B, Pinheiro RSN, Bitencourt FS, Farias CNF, D’Albuquerque LAC. Avaliação da qualidade de vida em pacientes cirróticos com hérnia da parede abdominal. ABCD Arq Bras Cir Dig 2009;22(4):222-5

RESUMO - Racional - As hérnias de parede abdominal são um problema frequente no paciente cirrótico. Elas costumam ser volumosas e muito sintomáticas. Objetivo - Avaliar a qualidade de vida, através da aplicação do questionário Short Form-36 (SF-36), nos pacientes com hérnia e cirrose. Métodos - Foram avaliados 39 pacientes cirróticos com hérnia de parede abdominal estratificados pela classificação de Child e submetidos ao questionário SF-36 por um mesmo profissional. Resultados - A média dos escores obtidos no SF-36 mostra diminuição em todos os domínios nos pacientes cirróticos, principalmente no aspecto físico. O grupo Child B mostrou pior avaliação ao estado geral de saúde e capacidade funcional em relação ao grupo Child A, contudo não houve diferença em relação ao aspecto físico. Conclusão - A presença de hérnia de parede abdominal ocasiona piora na qualidade de vida do paciente cirrótico. A deterioração da função hepática está relacionada a pior qualidade de vida. A hérnia abdominal leva a restrição importante no aspecto físico também no Child A. DESCRITORES - Cirrose. Hérnia. Qualidade de vida. Ascite.

\section{INTRODUÇÃO}

A hérnia de parede abdominal é problema frequente nos pacientes cirróticos. Sua incidência é bem mais elevada nesses pacientes, estando presente em mais de $20 \%$ dos que apresentam apenas cirrose ${ }^{1}$ e em até $40 \%$ dos pacientes que apresentam também ascite ${ }^{2}$. Existem diversos fatores nesses indivíduos que favorecerem o desenvolvimento da hérnia umbilical, como o aumento da pressão intra-abdominal devido à formação de ascite, maior fraqueza da aponeurose abdominal e redução da musculatura abdominal em consequência do estado nutricional deficiente, e pela contribuição da dilatação da veia umbilical recanalizada aumentando falhas pré-existentes na aponeurose peri umbilical ${ }^{11}$.

Essas hérnias tendem a adquirir maiores proporções tanto da falha aponeurótica como do volume protraído, principalmente nos pacientes com ascite ${ }^{2}$.O grande volume do saco herniário favorece a ocorrência de complicações, dentre elas as úlceras de pressão, ruptura da pele, extravasamento de líquido ascítico e propensão a peritonite bacteriana secundária ${ }^{2,9}$, além de causar fre-

Trabalho realizado no Serviço de Transplante de Órgãos do Aparelho Digestivo do Departamento de Gastroenterologia do Hospital das Clínicas da Faculdade de Medicina da Universidade de São Paulo, São Paulo, SP, Brasil

Endereço para correspondência: Wellington Andraus, e-mail: wellington@usp.br / wandraus@terra.com.br quentemente desconforto e dor, dificultando até mesmo o convívio social.

As hérnias inguinais também podem sofrer as consequências da pressão abdominal elevada pela presença da ascite adquirindo grandes dimensões, progredindo com frequência para a região escrotal, formando grandes hérnias inguino-escrotais. Além das complicações já mencionadas, provocam dificuldade à deambulação e prejuizo de qualidade de vida.

Os pacientes cirróticos apresentam complicações dependentes da função hepática como ascite, encefalopatia, hemorragia digestiva, dentre outras, e que levam a uma piora na qualidade de vida, e que na maioria das vezes só se resolve com o transplante de fígado. A hérnia de parede abdominal nestes pacientes provoca desconforto, alterações na deambulação, perfuração, e tem a possibilidade de resolução cirúrgica antes do transplante.

Este trabalho tem como objetivo avaliar a qualidade de vida de pacientes cirróticos com hérnias de parede abdominal.

\section{MÉTODOS}

Estudo prospectivo com a intenção de avaliar o déficit de qualidade de vida em pacientes cirróticos com hérnia de parede abdominal.

Foram analisados 39 pacientes consecutivos da uni- 
dade de Cirurgia e Transplante de Fígado, do Hospital das Clínicas da Faculdade de Medicina da Universidade São Paulo, com diagnóstico de cirrose hepática e hérnia de parede abdominal no período de agosto a dezembro de 2009. Esses pacientes foram submetidos à investigação de qualidade de vida através da aplicação do questionário SF-36 (Tabela 1).

TABELA 1 - Dados demográficos dos 39 pacientes cirróticos com hérnia de parede abdominal

\begin{tabular}{llll}
\hline & $\mathbf{n}$ & $\mathbf{\%}$ & \\
\hline Sexo & Masculino & 31 & $79 \%$ \\
& Feminino & 8 & $21 \%$ \\
\hline Idade & & $53,7(10,7)$ & \\
\hline Etiologia & álcool & 16 & $41 \%$ \\
& VHC & 7 & $18 \%$ \\
& Criptogênica & 6 & $15 \%$ \\
& VHB & 5 & $13 \%$ \\
& Budd Chiari & 2 & $5 \%$ \\
& outras & 3 & $8 \%$ \\
\hline MELD & & $11,9( \pm 3,6)$ & \\
\hline Child & A & 18 & $46 \%$ \\
& B & 20 & $51 \%$ \\
& C & 1 & $3 \%$ \\
\hline
\end{tabular}

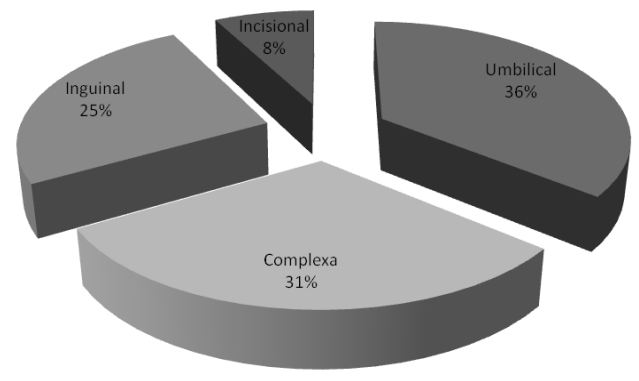

FIGURA 1 - Distribuição de hérnias de parede abdominal em pacientes cirróticos estudados

O questionário SF-36 (Medical Outcomes Study 36 Item short form health survey) é uma escala, já validada por outros estudos, que considera a percepção do indivíduo quanto ao seu próprio estado de saúde através de escores atribuídos a oito domínios da saúde: capacidade funcional, aspectos físicos, dor, estado geral de saúde, vitalidade, aspectos sociais, aspectos emocionais e saúde mental. Os dados são avaliados a partir da transformação das respostas em uma escala que varia de 0 a 100 para cada item, sendo 0 o pior possível e 100 o melhor.

Todos os pacientes responderam o questionário auxiliados por um mesmo profissional formado em psicologia.

A análise estatística foi realizada utilizando o programa Primer. Variáveis contínuas foram comparadas pelos métodos de t-Student para dados com distribuição normal. Foram considerados estatisticamente significativos valores de $\mathrm{P} \leq 0,05$.

\section{RESULTADOS}

O sexo masculino foi predominante com 31 homens e 9 mulheres. A idade média foi de 53,7 anos $( \pm 10,7)$. A causa predominante da cirrose foi alcoólica (41\%), seguida por VHC (18\%), criptogênica(15\%), VHB (13 $\%)$, Budd Chiari(5\%), outras (8\%). O MELD médio foi de $11,9( \pm 3,6)$ e a distribuição pelo Child foi de 18 pacientes $\mathrm{A}(46 \%), 20$ pacientes $\mathrm{B}(51 \%)$ e 1 paciente C $(3 \%)$.

Os tipos de hérnias identificadas na população estudada foram: umbilical em 23 pacientes, inguinal em 22 pacientes e incisional em três pacientes. Trinta e um porcento dos pacientes apresentavam mais de uma hérnia; esses casos foram classificados como hérnias complexas. Doze porcento dos pacientes possuíam hérnia inguinal bilateral e hérnia umbilical associada (Figuras 2, 4 e 5).

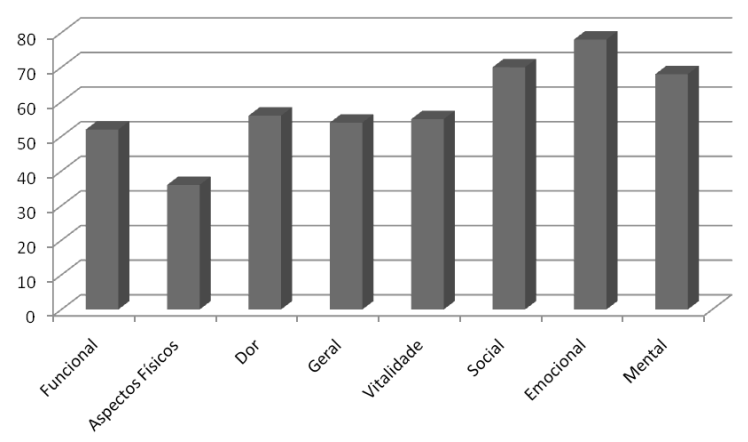

FIGURA 2 - Distribuição dos valores obtidos no questionário SF-36 entre os pacientes do estudo

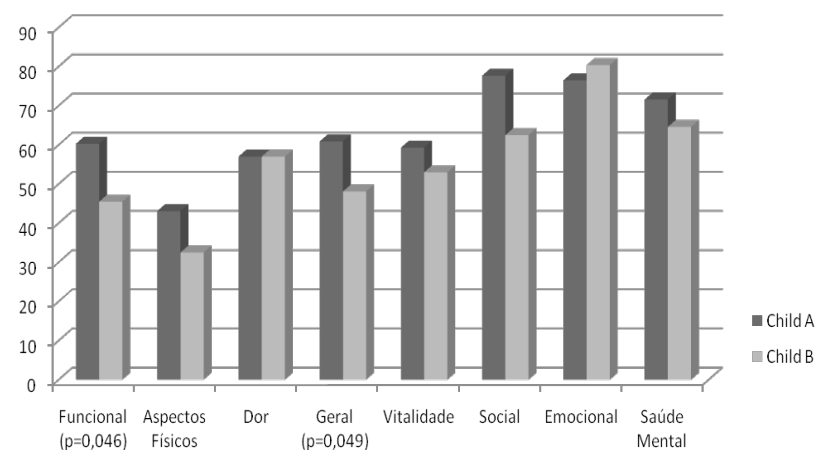

FIGURA 3 - Distribuição dos valores obtidos no questionário SF-36 entre os pacientes Child A e B

Os resultados obtidos pelo questionário SF-36 foram estratificados pela média global e dos pacientes Child A e $\mathrm{B}$, estando demonstrados na Figura 3. A análise estatística entre os resultados dos pacientes Child A e B demonstrou diferença significante $(\mathrm{P}<0,05)$ somente nos domínios de capacidade funcional e estado geral de saúde.

Os resultados do paciente Child C não foi computado, devido à pequena amostragem disponível. 


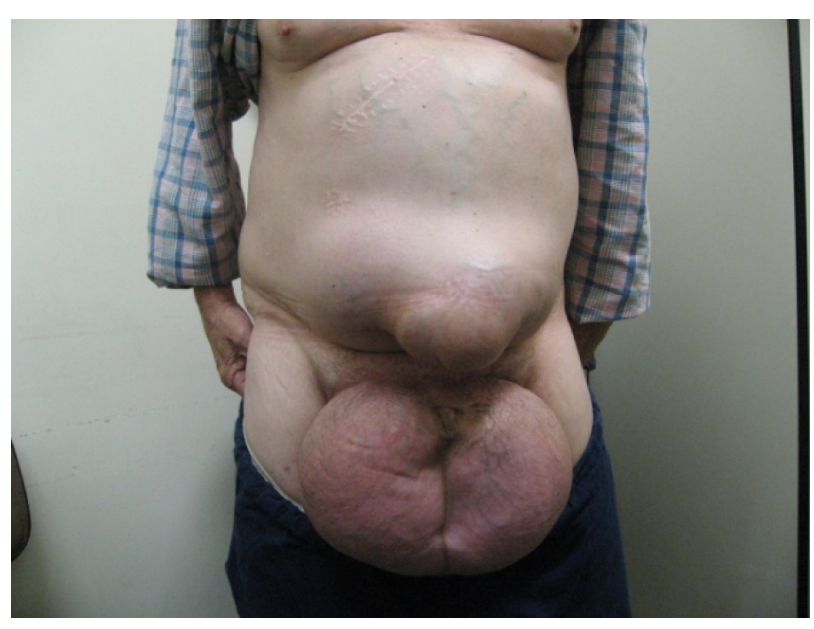

FIGURA 4 - Paciente com hérnia inguinal bilateral e umbilical de grande proporções

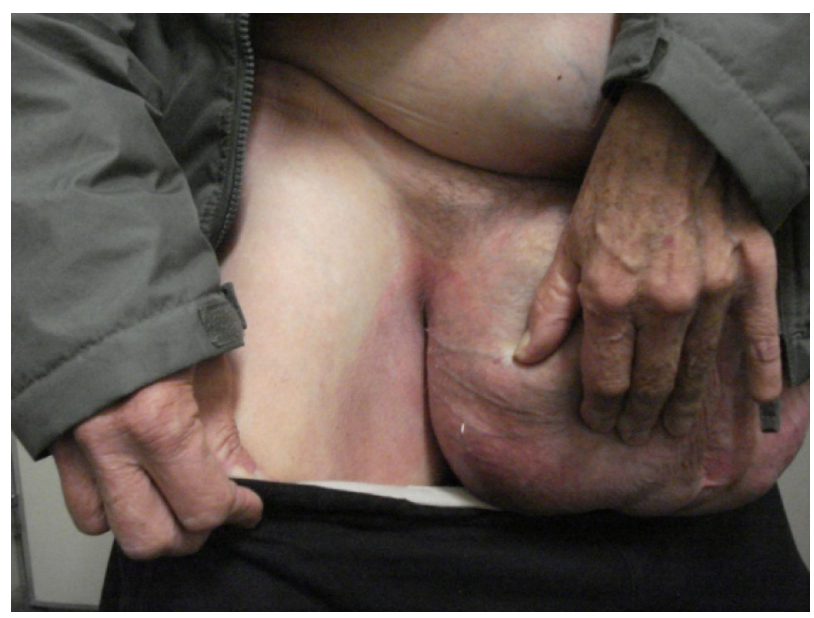

FIGURA 5 - Paciente com dermatite por friç̧ão do saco herniário escrotal

\section{DISCUSSÃO}

Os pacientes cirróticos, com a piora da função hepática, apresentam complicações como ascite volumosa, encefalopatia, desnutrição que dificultam as atividades habituais e levam a uma piora na qualidade de vida. $\mathrm{O}$ tratamento definitivo para estas complicações é o transplante de fígado, que devido à grande fila de espera pode ser demorado. Por outro lado, o desenvolvimento de hérnia de parede abdominal em pacientes cirróticos é muito frequente, e estas hérnias são passíveis de correção mesmo antes do transplante.

Ascite persistente está relacionada com hérnia de parede abdominal em pacientes cirróticos devido ao aumento da pressão intra-abdominal e à fraqueza da parede abdominal destes pacientes ${ }^{1}$. Quando a ascite está presente, as hérnias são de difícil tratamento e com tendência a complicações. Com o tempo, elas podem atingir dimensões impression- antes, tornando sua correção às vezes complicada ${ }^{6,7}$.

A correção eletiva para as hérnias em pacientes cirróticos é frequentemente desencorajada devido a alta morbidade e mortalidade relacionados ao procedimento e também à elevada taxa de recidiva ${ }^{4}$. Contudo, a literatura mais recente mostra que a morbidade e mortalidade para as operações eletivas de hérnias é menor quando comparadas com as operações de urgência ${ }^{5,9}$. Assim sendo, alguns autores acreditam que a correção cirúrgica eletiva, com prepare pré-operatório adequado, em centros com experiência, está indicado nestes pacientes $\mathbf{s}^{3,4,9}$.

Patti et al. ${ }^{10}$ avaliaram 32 pacientes cirróticos com hérnia inguinal aplicando o questionário SF-36 antes e após a correção cirúrgica e encontraram melhora em oito domínios do questionário com a operação, concluindo que houve melhora significativa na qualidade de vida destes pacientes. Este mesmo questionário foi usado anteriormente por Lawrence et al. ${ }^{8}$ na avaliação de 140 pacientes não cirróticos submetidos à correção de hérnia inguinal e observaram melhora na qualidade de vida dos pacientes submetidos à operação tanto por via aberta como laparoscópica.

No presente trabalho, os pacientes avaliados indicaram a limitação por aspecto físico como sendo o pior domínio no questionário aplicado, mostrando a importância da hérnia na piora da qualidade de vida nestes pacientes. Um dos principais aspectos apontados foi a dificuldade de retorno ao trabalho devido à presença de herniação. Os pacientes com ascite podem apresentar aumento expressivo do saco herniário, dor mais importante e dificuldade para deambular, aumentando ainda mais a restrição física e o convívio social.

Na comparação entre os pacientes com Child A e com Child B houve piora significativa no estado geral de saúde e capacidade funcional no grupo de Child B; no entanto, não houve diferença significativa no domínio aspecto físico do questionário entre estes dois grupos, apesar deste quesito (aspecto físico) ser o pior avaliado por todos os pacientes. Estes dados demonstram que a piora da função hepática leva a uma piora da qualidade de vida, por outro lado, a simples presença de hérnia no grupo Child A leva a uma piora na qualidade de vida destes pacientes, principalmente no aspecto de restrição física, onde não foi observada diferença significativa com o grupo Child $\mathrm{B}$.

Há necessidade de trabalhos com maiores casuísticas e também comparativos com pacientes já submetidos à correção cirúrgica para avaliação mais criteriosa da qualidade de vida neste grupo de pacientes.

\section{CONCLUSÃO}

A presença de hérnia de parede abdominal leva à piora na qualidade de vida do paciente cirrótico, principalmente em relação ao aspecto físico. 
Andraus W, Paoletti B, Pinheiro RSN, Bitencourt FS, Farias CNF, D’Albuquerque LAC. Quality of life in patients with abdominal hernia and cirrhosis. ABCD Arq Bras Cir Dig 2009;22(4):222-5

ABSTRACT - Background - Abdominal hernias are frequent issue in cirrhotic patients. These hernias tend to be large and symptomatic. Aim - To analyze the quality of life using a Short Form-36 (SF-36) questionnaire in cirrhotic patients with abdominal hernia. Methods - Thirty nine cirrhotic patients with abdominal wall hernia were analyzed. They were stratified according to Child's classification and answered the SF-36 questionnaire administered by a same professional. Results - The scores from SF-36 show impairment in all questionnaire domains in cirrhotic patients, especially in the physical aspect. The Child B group showed worse health general condition and functional capacity comparing with Child A group; however it did not show any significant difference related to physical aspect. Conclusion - The presence of abdominal hernia leads to worse quality of life in cirrhotic patients. Hepatic function deterioration is related to a worse quality of life. Abdominal hernia causes an important restriction in physical aspect even in Child A.

HEADINGS - Cirrhosis. Hernia. Quality of life. Ascites.

\section{REFERÊNCIAS}

1. Belghiti J, Durand F. Abdominal wall hernias in the setting of cirrhosis. Semin Liver Dis. 1997;17(3):219-26.

2. Carbonell AM, Wolfe LG, DeMaria EJ. Poor outcomes in cirrhosis-associated hernia repair: a nationwide cohort study of 32,033 patients. Hernia. 2005 Dec;9(4):353-7.

3. Shlomovitz E, Quan D, Etemad-Rezai R, McAlister VC. Association of recanalization of the left umbilical vein with umbilical hernia in patients with liver disease. Liver Transpl. 2005 Oct;11(10):1298-9.

4. Marsman HA, Heisterkamp J, Halm JA, Tilanus HW, Metselaar HJ, Kazemier G. Management in patients with liver cirrhosis and an umbilical hernia. Surgery. 2007 Sep;142(3):372-5.

5. Kirkpatrick $S$, Schubert T. Umbilical hernia rupture in cirrhotics with ascites. Dig Dis Sci. 1988 Jun;33(6):762-5.

6. Kaya M, Yucesan S. Spontaneous ruptured umbilical hernia in a child with hepatic cirrhosis and ascites. Pediatr Surg Int. 2005 May;21(5):413-4.
7. Hansen JB, Thulstrup AM, Vilstup H, Sorensen HT. Danish nationwide cohort study of postoperative death in patients with liver cirrhosis undergoing hernia repair. Br J Surg. 2002 Jun;89(6):805-6.

8. Hurst RD, Butler BN, Soybel DI, Wright HK. Management of groin hernias in patients with ascites. Ann Surg. 1992 Dec;216(6):696-700.

9. Fagan SP, Awad SS, Berger DH. Management of complicated umbilical hernias in patients with end-stage liver disease and refractory ascites. Surgery. 2004 Jun;135(6):679-82.

10. Patti R, Almasio PL, Buscemi S, Fama F, Craxi A, Di Vita G. Inguinal hernioplasty improves the quality of life in patients with cirrhosis. Am J Surg. 2008 Sep;196(3):373-8.

11. Lawrence K, McWhinnie D, Jenkinson C, Coulter A. Quality of life in patients undergoing inguinal hernia repair. Ann R Coll Surg Engl. 1997 Jan;79(1):40-5.

Fonte de financiamento: não há Conflito de interesse: não há Recebido para publicação:27/06/2009 Aceito para publicação: 14/10/2009 